Purdue University

Purdue e-Pubs

ASEE IL-IN Section Conference

\title{
Tethered Picosatellites: A First Step towards Electrodynamic Orbital Control and Power Generation
}

William A. Bauson

Taylor University

Follow this and additional works at: https://docs.lib.purdue.edu/aseeil-insectionconference

Bauson, William A., "Tethered Picosatellites: A First Step towards Electrodynamic Orbital Control and Power Generation" (2018). ASEE IL-IN Section Conference. 1.

https://docs.lib.purdue.edu/aseeil-insectionconference/2018/tech/1

This document has been made available through Purdue e-Pubs, a service of the Purdue University Libraries. Please contact epubs@purdue.edu for additional information. 


\title{
Tethered Picosatellites: A First Step towards Electrodynamic Orbital Control and Power Generation
}

\author{
William A. Bauson \\ Taylor University
}

\begin{abstract}
University students routinely design and launch small satellites into space, giving students the opportunity to gain experience in a wide variety of STEM disciplines. This paper describes work in progress on one such project, "MagnITO-Sat," which consists of two picosatellites connected by a conductive tether. The ultimate aim of the tether is to provide electrodynamic thrust generation and power generation. This project will test three major components of the system: 1) the tether deployment mechanism; 2) the highvoltage biasing to enable current flow through a "phantom loop" formed by the conductive tether and the ionosphere; and 3) an optical (near-infrared) link that provides communication between the two picosatellites. A Globalstar radio transmits data and measurements to the ground.
\end{abstract}

\subsection{Introduction}

Taylor University's Senior Engineering Project engages engineering students in learning and applying systems design techniques to real-world projects. Previous projects include the design of three different satellites, one of which flew in Low Earth Orbit in 2014. The current project is the Magnetically Induced Tethered Orbit adjustment Satellite (MagnITO-Sat), funded by the Indiana Space Grant Consortium. We are developing MagnITO-Sat as part of the ET-Sat program, a collaboration between Twiggs Space Lab, Virginia Space, NASA Wallops Flight Facility, Orbital ATK, and Near Space Launch.

MagnITO-Sat consists of two small satellites connected by a conductive tether. The ultimate aim of the tether is provide electrodynamic thrust generation and power generation. This project's mission is to test three major components of the system: 1) the tether deployment mechanism, 2) the high-voltage biasing to enable current flow through a "phantom loop" formed by the conductive tether and the ionosphere, and 3) an optical (near-infrared) link that provides communication between the two picosatellites. A Globalstar radio transmits measurement data to the ground.

Taylor University students developed the MagnITO-Sat concept for the 2016-2017 Senior Engineering Project. The project included drop testing to observe tether deployment in a simulated low-gravity environment. Over the summer of 2017, another group of students began hardware and software design. The team will complete the design during the 2017-2018 Senior Project. High altitude balloon testing, vibration testing, and thermal-vacuum testing will take place during the spring of 2018. As part of the ET-Sat project, the satellites will launch from the second stage of an Orbital ATK rocket in October 2018 on a Space Station resupply mission. The second stage launch will be an industry first. 
The ET-Sat satellites will fly at an altitude of approximately $200 \mathrm{~km}$. Because of the atmospheric drag at this altitude, they will stay in orbit for a maximum of two weeks before burning up in the Earth's atmosphere.

\subsection{Tethers in Space}

Tethers have many potential uses in space, including: changing orbit through momentum exchange [1], satellite deorbiting through atmospheric drag [2], space elevators [3], power generation [1], and thrust generation [1].

Mario Grossi, of the Smithsonian Astrophysical Observatory, and Giuseppe Colombo of Padua University proposed using tethers in space in the early 70's [1]. This ultimately resulted in experiments on NASA's STS-46 (1992) and STS-75 (1996) Space Shuttle missions [1].

STS-46 used a 286-meter long strand of insulated copper wire to tether a satellite to the Space Shuttle. The Space Shuttle deployed a satellite 286 meters directly above the Shuttle. The satellite remained in this position for 20 hours, stabilized by the gravity gradient between the Shuttle and satellite [1].

In STS-75, the Shuttle deployed a tethered satellite to a distance of $19.7 \mathrm{~km}$ above the Shuttle. After five hours of operation, the tether broke. The experiment was able to provide useful data on electrodynamic tether operation before the break occurred [1].

\subsection{Electrodynamic Tethers}

Taylor University's current satellite project focuses on electrodynamic tethers. Electrodynamic tethers provide power generation and thrust generation. Through power generation, the tether can recharge a spacecraft's batteries simply by flying through space. With thrust generation, the tether can change a spacecraft's orbit by pushing the spacecraft to speed it up or by providing drag to slow it down.

The electrodynamic tether operates on the same principles as the common electric generator and the electric motor. According to Faraday's laws of electromagnetic induction, whenever a conductor is placed in a varying magnetic field (or a conductor is moved in a magnetic field), an emf (electromotive force or voltage) is induced in the conductor. This is the working principle of an electric generator. In the case of a satellite, a conducting tether moves through the Earth's magnetic field, inducing a voltage across the tether. The induced voltage can provide power for the satellite.

Conversely, whenever a current-carrying conductor is in a magnetic field, it experiences a mechanical force. This is the working principle of an electric motor. In the case of a satellite, a current flows through a conducting tether, resulting in a mechanical force on the tether. The resulting force can provide thrust for the satellite. 
Thus, by driving a current through an electrodynamic tether, a magnetic force can be generated which opposes the Earth's magnetic field, thereby changing the nanosatellite's orbit.

Additionally, the tether can generate power as it travels through the Earth's magnetic field (Figure 1).

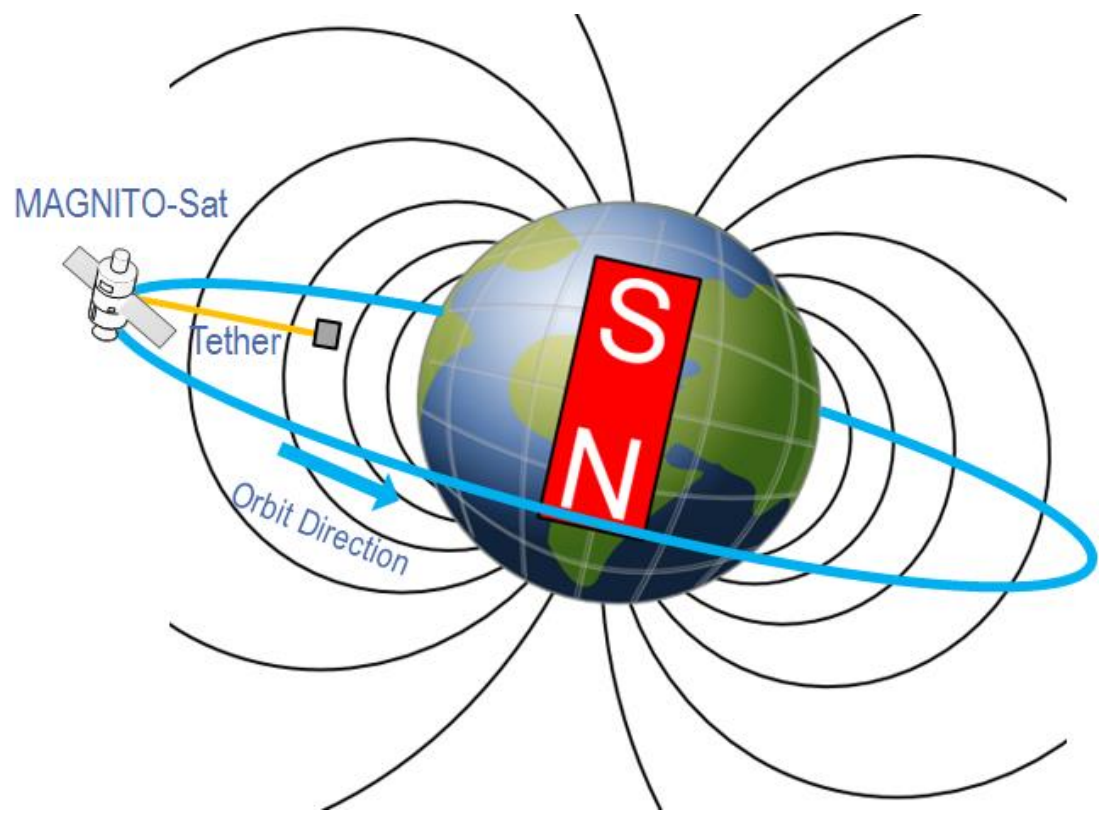

Figure 1. Electrodynamic Tether Action.

The force generated by a straight powered tether is given by

$$
\vec{F}=(\overrightarrow{I L} \times \vec{B})
$$

where $I=$ current flowing through the tether

$L=$ length of the tether

$B=$ magnetic field strength

Equation (1) can also be written as

$$
F=I L B \sin \theta
$$

where $\theta=$ angle between $\vec{L}$ and $\vec{B}$

When the tether is straight and perpedicular to the magnetic field lines along its length, the equation for the voltage induced along the tether is

$$
V=(\vec{v} \times \vec{B}) \cdot \vec{L}
$$


where $\mathrm{V}=$ induced voltage across the tether length

$\vec{v}=$ tether velocity relative to the geomagnetic field

$\vec{B}=$ magnetic field strength

$\vec{L}=$ tether length pointing the direction of positive current flow

\subsection{The MagnITO-Sat Project}

The MagnITO-Sat development program is a multi-year project funded by the Indiana Space Grant Consortium (Figure 2). The project offers students experience in systems engineering, teamwork, research, collaboration, and many other areas that will benefit their future careers.

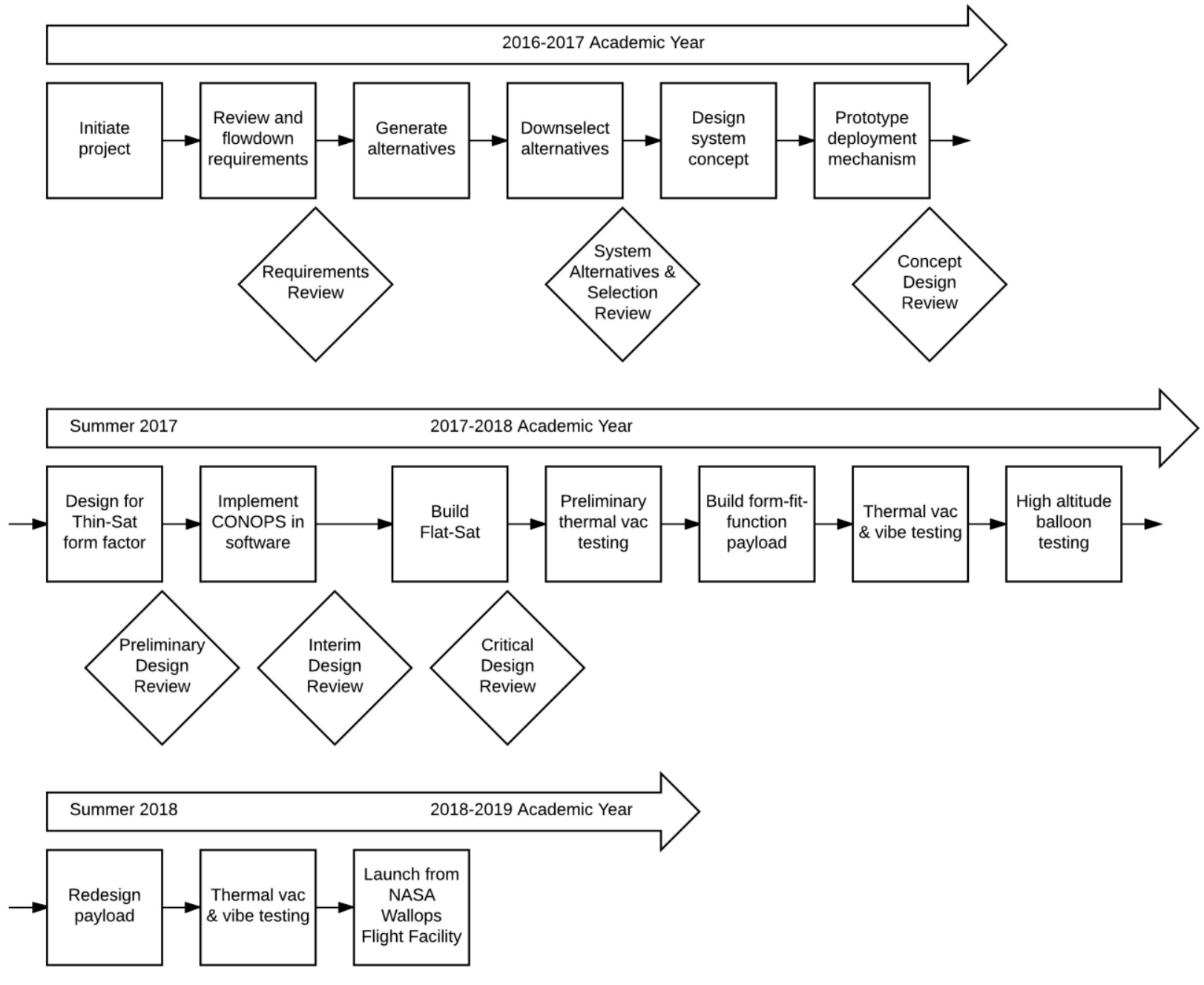

Figure 2. MagnITO-Sat Project Layout. 


\subsection{6-2017 Academic Year Activities}

The project started in the fall of 2016 as an Engineering Senior Capstone. The goal was to design and prototype a $2 \mathrm{U}$ or $3 \mathrm{U}$ CubeSat ${ }^{1}$ that would investigate space tethers in the Low Earth Orbit (LEO) region. A rigorous Systems Engineering Process began with determining requirements, then analyzing system and subsystem requirements, resulting in a Requirements Review.

Next, the team performed trade studies and modeling to generate several alternatives. The team selected a single concept and presented it at a System Alternatives and Selection Review. For Concept Design, the team developed the ConOps and software diagrams, selected a bus, developed the tether deployment concept, and built a 3D model of the satellite. The team also designed and built a prototype tether deployment mechanism and microgravity test unit. They performed various microgravity drop tests on the deployment mechanism. Over the course of the project, the team performed literature searches, performed detailed technical analyses, and held discussions with scientists on the forefront of space tether research from Naval Research Labs, the University of Michigan, and Tethers Unlimited Inc. Faculty members and outside parties attended the various design reviews. Feedback to the team helped guide changes to the design concepts.

\subsection{Summer 2017 Activities}

Near the end of the 2016-2017 academic year, Taylor University received an opportunity to participate in the ET-Sat program, a collaboration between Twiggs Space Lab, Virginia Space, NASA Wallops Flight Facility, Orbital ATK, and Near Space Launch. This program will allow us to test some of the key MagnITO-Sat concepts in a picosatellite form factor called "ThinSat." The ThinSat is a $111 \mathrm{~mm} \times 114 \mathrm{~mm} \times 12.5 \mathrm{~mm}$ picosatellite that comes pre-loaded with a power supply and Globalstar radio. The university provides a $52 \mathrm{~mm} \times 108 \mathrm{~mm}$ payload board that fits into half of the ThinSat volume. The payload sends data to the ThinSat motherboard, which periodically transmits data to the Globalstar communications satellite network. The ET-Sat program has a launch scheduled for October 2018.

During the summer, a team of five students began designing and testing the major MagnITO-Sat subsystems. The work included continued communication with experts in the field to help guide the design. The summer included a trip to Morehead State University to tour their Space Science and nanosatellite testing facilities.

\subsection{7-2018 Academic Year and Beyond}

In the fall of the 2017-2018 academic year, a team of 10 students continued developing the MagnITO-Sat subsystems for the ThinSat. At this writing, the team is finishing a Flat-Sat system bench that incorporates all of the major subsystems and preliminary operating software.

The rest of the academic year will be spent designing the MagnITO-Sat subsystems into the final payload form factor and performing thermal vacuum and vibration testing. Final testing and

\footnotetext{
${ }^{1}$ The standard (1U) CubeSat is $10 \times 10 \times 11.35 \mathrm{~cm}$ in size. A 3U CubeSat stacks three $1 \mathrm{U}$ CubeSats lengthwise.
} 
redesign will take place during the summer. We will deliver the final ThinSat to Twiggs Space Lab in the summer of 2018 for the launch in October 2018.

\subsection{Pedagogy}

Working in teams similar to those found in industry, students started with a provided requirements document, flowed down the requirements, generated alternative concepts, downselected concepts, and finally designed and prototyped the various satellite subsystems. A major part of the effort required students to develop mathematical models for surface area drag, power usage, tether thrust, etc. Teams include students from several of Taylor's majors: Computer Science, Engineering (mechanical and electrical concentrations), and Computer Engineering.

Student leaders receive common industry titles (Program Manager, Chief Engineer, etc.) and are expected to perform the tasks required of those jobs. Classes typically meet three times per week. During the class, the Program Manager gets status reports from each student, then sets the direction for next steps. After the instructor provides any additional direction or teaching, the team is released to work on their part of the project. Students are expected to put in an additional two hours per week for each credit hour of the class. During this time, the students typically work together, helping each other with designs, analysis, etc. Professors from the Computer Science and Physics and Engineering departments serve as consultants to the students.

Throughout the project, instructors taught the students how to create proper documentation and follow systems design procedures. Specific technical direction took place one-on-one. In several cases, industry experts participated in conference calls with the students and instructors to provide assistance to the team. Grading is based on each student's trade studies and analysis documentation, engineering logbooks, and presentations.

Learning was also encouraged through participation in the SmallSat Conference for small satellites in Logan, Utah, as well as through trips to Morehead State University's Space Science facility and Purdue University's nanotechnology facility.

\subsection{System Overview}

\subsection{The Mission}

The current ThinSat mission is to test three major components of the original MagnITO-Sat concept: 1) the tether deployment mechanism, 2) the high-voltage biasing to enable current flow through a "phantom loop" formed by the conductive tether and the ionosphere, and 3) an optical (near-infrared) link that provides communication between the two picosatellites. Additional sensors will be included to measure other related space parameters.

\subsection{Phantom Loop}

MagnITO-Sat consists of two satellite bodies connected by a conductive tether. A key element of the system is the Phantom Loop (Figure 3). In order for current to flow through the 
conductive tether, there must be a closed loop. One solution for closing the loop would be to use two parallel wires for the tether. However, the magnetic fields caused by the two parallel wires would cancel each other out. Instead, a "phantom path" closes the loop. One satellite body contains an electron emitter (thermionic emitter) while the other satellite body contains an electron collector. As the emitter emits electrons, the collector collects free electrons from the ionosphere. This "phantom path" closes the current loop, allowing current to flow through the single tether wire.

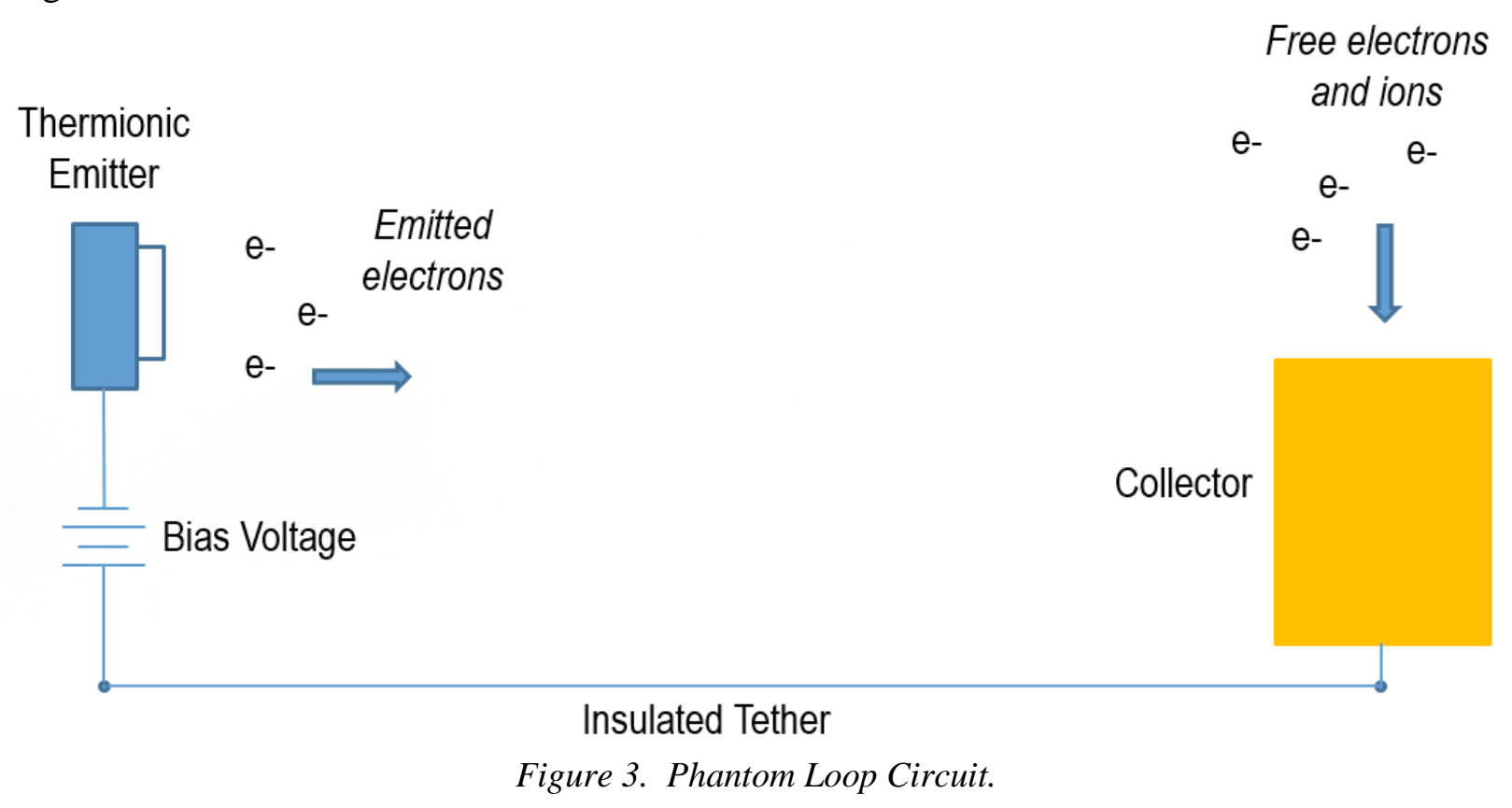

\subsection{MagnITO-Sat and ThinSat System Requirements}

Both the MagnITO-Sat and ThinSat designs started from high-level system requirements such as physical size, electrical power, functional requirements, etc. Appendix A contains the MagnITO-Sat requirements. Appendix B shows the ThinSat requirements. These high-level requirements were flowed down to specific subsystem requirements.

\subsection{MagnITO-Sat System Design}

The primary direction for the project has been to focus on thrust generation rather than power generation. Several models (Table 1) helped determine the amount of thrust required to affect the nanosatellite's orbit, the tether length, tether voltage, tether current, etc. 
Table 1. Models Used in the Design Phase.

\begin{tabular}{|l|l|}
\hline Model & Comments \\
\hline Surface area drag model & $\begin{array}{l}\text { Used to determine atmospheric drag on the satellite and } \\
\text { tether. Based on atmospheric density at altitude, surface } \\
\text { area, and tether length. }\end{array}$ \\
\hline Power usage model & $\begin{array}{l}\text { Used to determine amount of power available for the } \\
\text { satellite. Based on number of illuminated solar cells } \\
\text { and diurnal analysis to estimate amount of solar energy } \\
\text { in orbit. }\end{array}$ \\
\hline Orbital lifetime model & $\begin{array}{l}\text { Used to estimate B field along the orbit. Based on } \\
\text { NOAA magnetic field calculators. }\end{array}$ \\
\hline Tether thrust model & $\begin{array}{l}\text { Used to estimate number of days in orbit. Based on } \\
\text { inclination, altitude, drag, and thrust. }\end{array}$ \\
\hline $\begin{array}{l}\text { Used to determine thrust generated by the tether. Based } \\
\text { on drag, ionosphere, plasma contactor resistance, } \\
\text { altitude, inclination, geomagnetic field. }\end{array}$ \\
\hline
\end{tabular}

The original MagnITO-Sat design used a 3U CubeSat physical package. The result of combining the analyses of the various models showed that, for a $3 \mathrm{U}$ CubeSat:

- To make up for atmospheric drag caused by the satellite, tether, and end mass, we need at least $2 \mu \mathrm{N}$ of thrust.

- The tether must be approximately 150 meters long.

- The tether can be made of 30-gauge insulated copper wire.

- The tether current must be at least $2 \mathrm{~mA}$.

- Voltage applied to the tether must be approximately 220 volts.

- The net thrust will be approximately $3.6 \mu \mathrm{N}$.

- The power consumed by operating the tether for thrust generation will be approximately 1.5 watts.

- The tether should operate on at least a $40 \%$ duty cycle to overcome drag.

\subsection{U MagnITO-Sat Concept}

Figure 4 shows the functional block diagram for the 3U MagnITO-Sat. The satellite would contain all the electronics needed to power the system, a processor to control operations, a Globalstar radio to communicate with the ground, an attitude control system, and a tether deployment mechanism (Figure 5). 


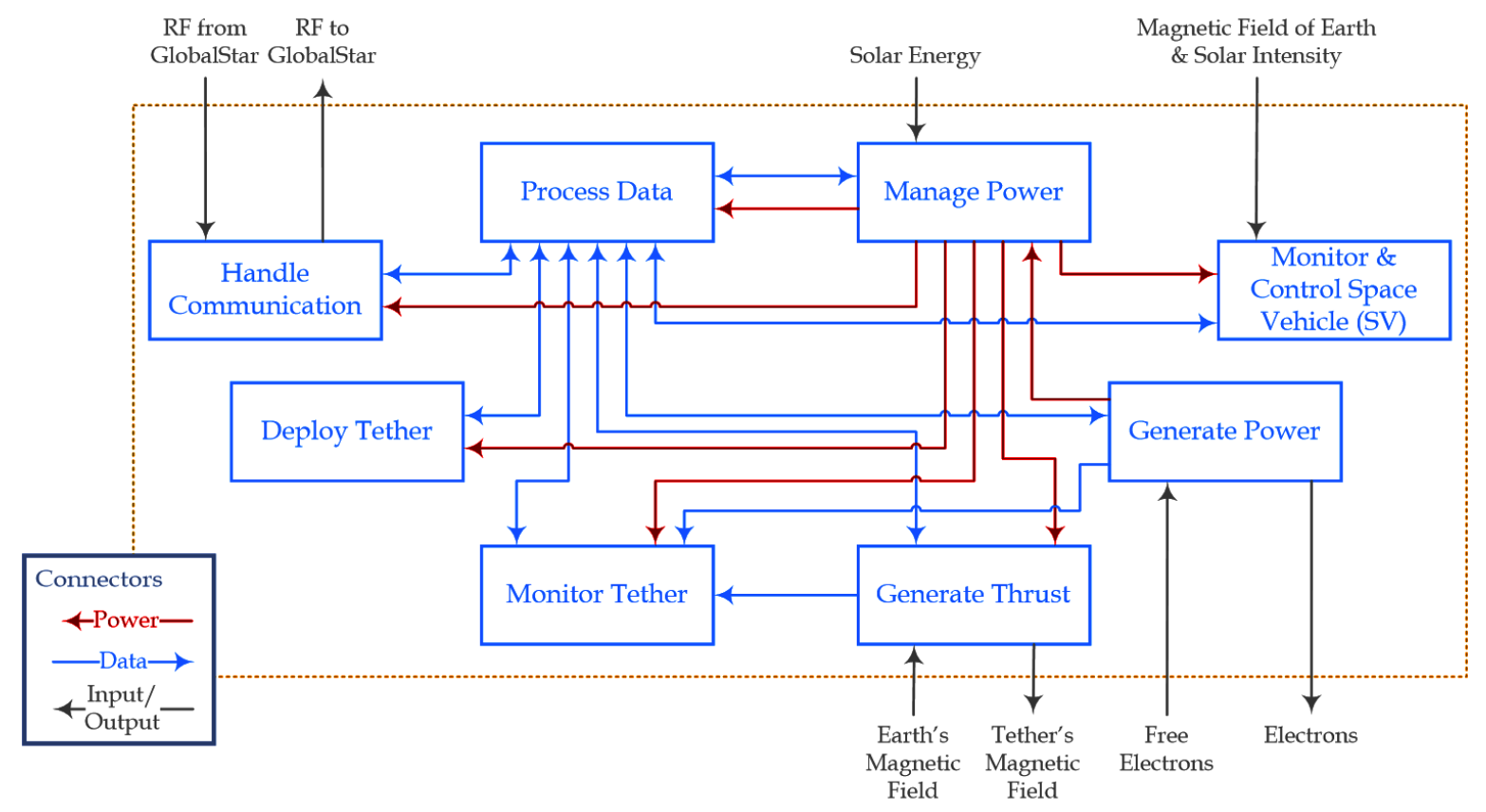

Figure 4. 3U MagnITO-Sat Functional Block Diagram.

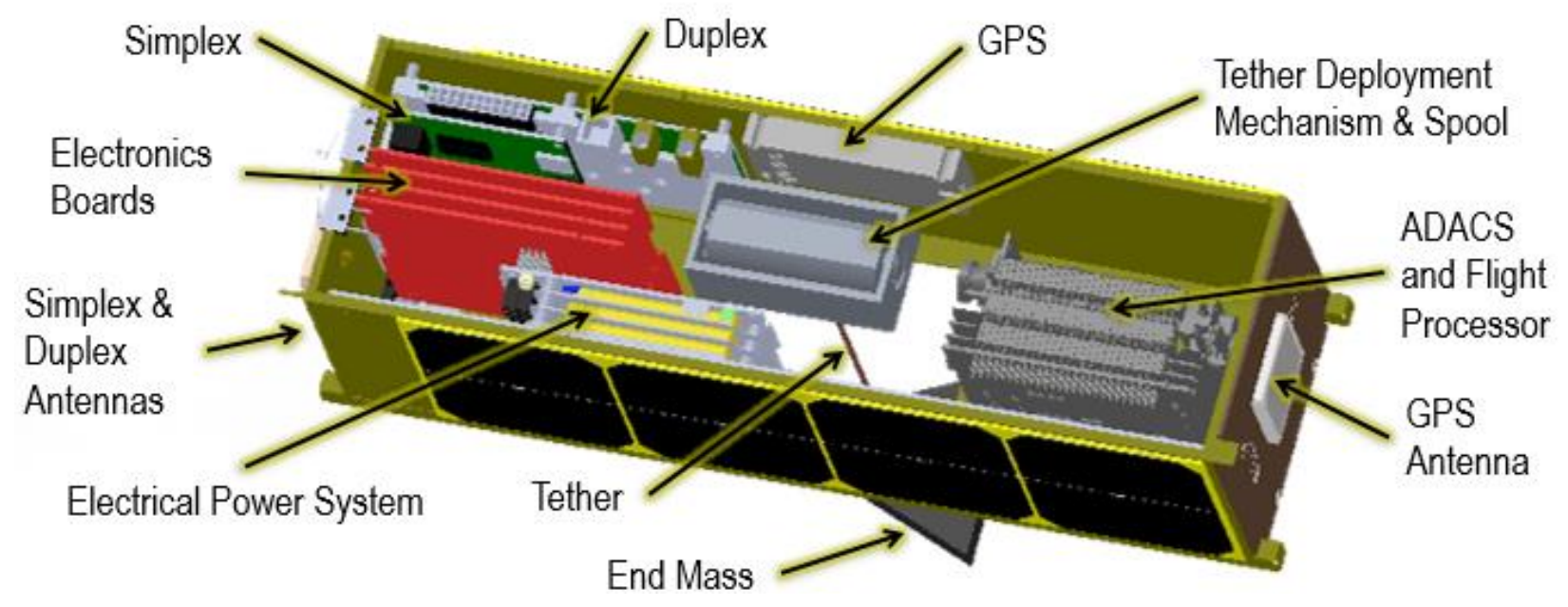

Figure 5. 3U MagnITO-Sat Concept.

\subsection{U MagnITO-Sat Concept of Operations (ConOps)}

Figure 6 shows the various modes of operation. The Concept of Operations (ConOps) is as follows:

- Background Operations (after launch separation)

- Solar Charging

- Health \& Safety Monitoring

- Commissioning Phase

○ Launch Vehicle (LV) Separation Mode: first 15 minutes following LV separation 
- Power on On-Board Computer

- RF Silence

- No maneuvering

- Detumble Mode I: after 15 minutes in Launch Vehicle Separation Mode

O RF Silence: first 30 minutes

○ Actively de-tumble

- Goal: Establish communication with Globalstar (Simplex)

- Space Vehicle (SV) Zenith within $\pm 15^{\circ}$ of true Zenith

- Mission Phase: after communication with Globalstar is established in Detumble Mode I

- Detumble Mode II

- Goal: achieve complete attitude control

- Roll rate $\leq 1^{\circ}$ per minute

- SV Zenith within $\pm 10^{\circ}$ of true Zenith

- "Reset" mode of Mission Phase

- Allow for communications to and from Ground (Duplex)

○ Tether Monitoring Mode

- After deployment of tether, SV waits for minimum 3 orbits before running other operations (generating thrust or power)

- Track mission tether operations

- Monitor tether and other conditions before continuing with any tether operation

- Decide which tether operation is next: deploy tether, generate thrust, or generate power

○ Tether Deployment Mode I

- Deploy tether in increments, starting with 10 meters

- Return to Detumble Mode II

- Thrust Generation Mode

- Send current through wire to generate tether's magnetic field for three minutes (to be reviewed)

- Discontinue thrust generation before returning to Detumble Mode II

- Power Generation Mode

- Monitor power that the tether generates as it moves through the ionosphere

- Only operated under $300 \mathrm{~km}$ altitude

- Return to Detumble Mode II

- Override Modes: automatic interrupts during Mission Phase

○ Ground Override Mode

- Triggered by ground override command

- Waits for and executes commands from Ground

- Only other operations: background operations

○ Power Saver Mode: $60-80 \%$ battery

- Power down subsystems while recharging battery

- Data transmission via Simplex, only data receiving over Duplex

○ Low Power Mode: 40-60\% battery

- Power down subsystems while recharging battery

- Minimal data transmission via Simplex, only data receiving over Duplex 
○ Peril Power Mode: $\leq 40 \%$ battery

- Power down subsystems while recharging battery

- Data transmission over Simplex Beacon only

- Malfunctioning Parts Mode

- Malfunctioning or overheating part(s), or error message from part(s)

- Power part(s) off and on again

- Decommissioning Phase: $\leq 200 \mathrm{~km}$ altitude, preparing for rapid deorbit and burn-up

○ Tether Deployment Mode II

- Deploy tether to full length if possible (or if not already fully deployed)

- Run thrust (or power) generation \& gather related data

$\circ$ Decommissioning Phase

- Collect data measurements and send data to Ground as much and as frequently as possible

- Deorbit / burn-up

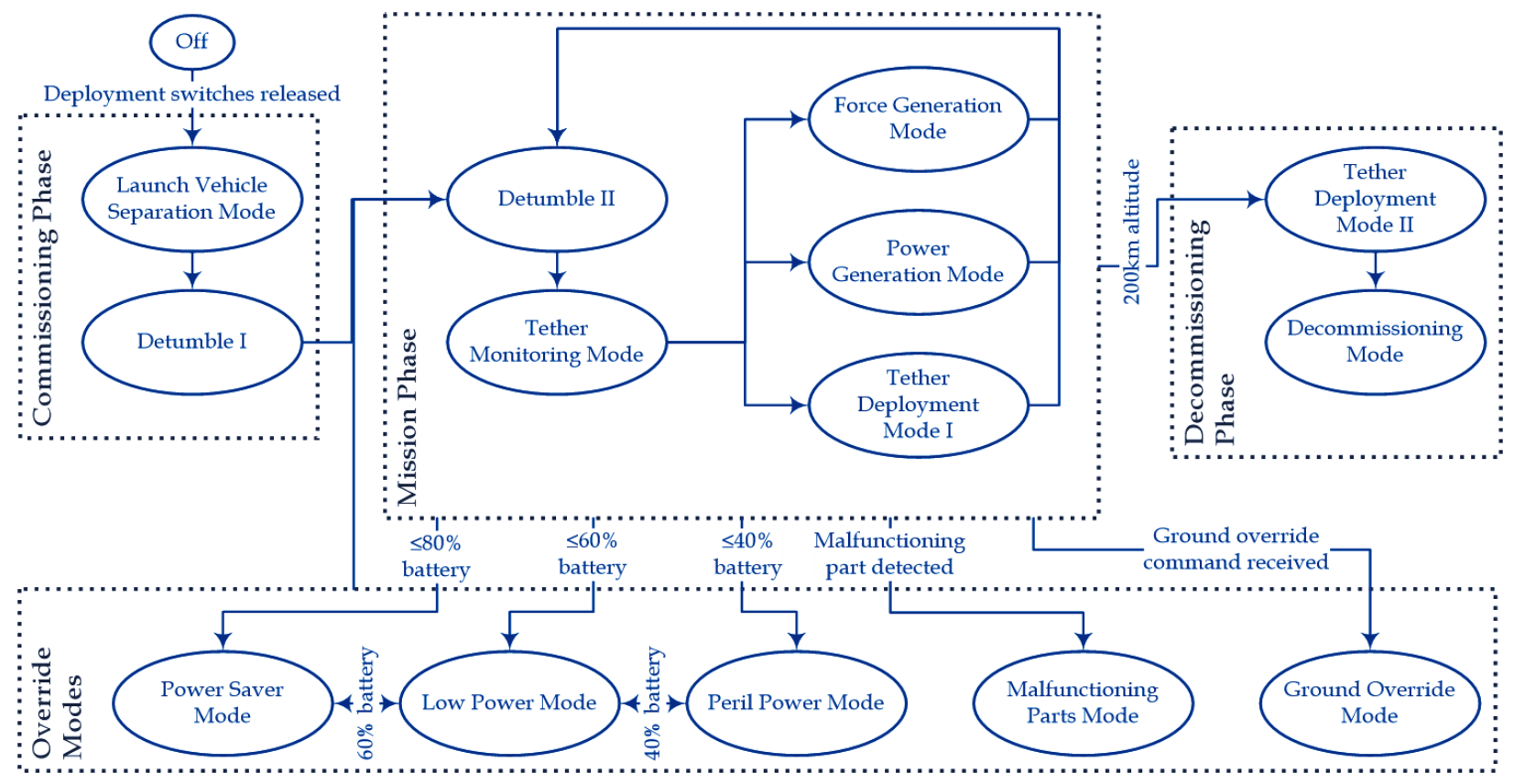

Figure 6. MagnITO-Sat Mode Diagram.

\subsection{ThinSat System Design}

As mentioned above, the ET-Sat program presented an opportunity to test some of the key MagnITO-Sat concepts in a picosatellite form factor called "ThinSat." The ThinSat is a $111 \mathrm{~mm}$ x $114 \mathrm{~mm}$ x $12.5 \mathrm{~mm}$ picosatellite that comes pre-loaded with a power supply and Globalstar radio. The university provides a $52 \mathrm{~mm} \times 108 \mathrm{~mm}$ payload board that fits into half of the ThinSat volume. The payload sends data to the ThinSat motherboard, which periodically transmits data to the Globalstar communications satellite network. 
The current ThinSat mission is to test three major components of the original MagnITO-Sat concept: 1) the tether deployment mechanism, 2) the high-voltage biasing to enable current flow through a "phantom loop" formed by the conductive tether and the ionosphere, and 3) an optical (near-infrared) link that provides communication between the two picosatellites. Additional sensors will be included to measure other related space parameters.

Table 2 shows the payload content to achieve these measurements. A Globalstar radio will transmit measurement data to the ground.

Table 2. Payload Content.

\begin{tabular}{|l|l|}
\hline Content & Purpose \\
\hline Thermionic emitter & Test phantom loop current generation \\
\hline Phantom loop high voltage supply & Test phantom loop biasing \\
\hline Deployment mechanism & Test tether deployment \\
\hline NIR data link & Communicate between the two ThinSats \\
\hline Inertial Measurement Unit (IMU) & Measure ThinSat dynamics \\
\hline Magnetic field sensor & Understand magnetic field strength for Lorenz force \\
\hline Electric field sensor & General science \\
\hline Temperature and light sensors & General science \\
\hline
\end{tabular}

\subsection{ThinSat Concept}

Figure 7 shows the system concept. A deployable 10-meter insulated tether will connect two ThinSats together. A mothership ThinSat contains a duplex Globalstar radio to receive commands from the ground and send data to the ground. An "accordion" structure composed of printed circuit boards provides the dual function of passing control signals between the mothership and ThinSat-1 and serving as the electron collector for the phantom loop. A near infrared (NIR) link provides communication between ThinSat-1 and ThinSat-2. ThinSat-1 contains the tether deployment mechanism. ThinSat-2 contains the phantom loop control electronics and the various science sensors. Figure 8 is a mockup of the mothership, ThinSat- 1 , and the accordion. 


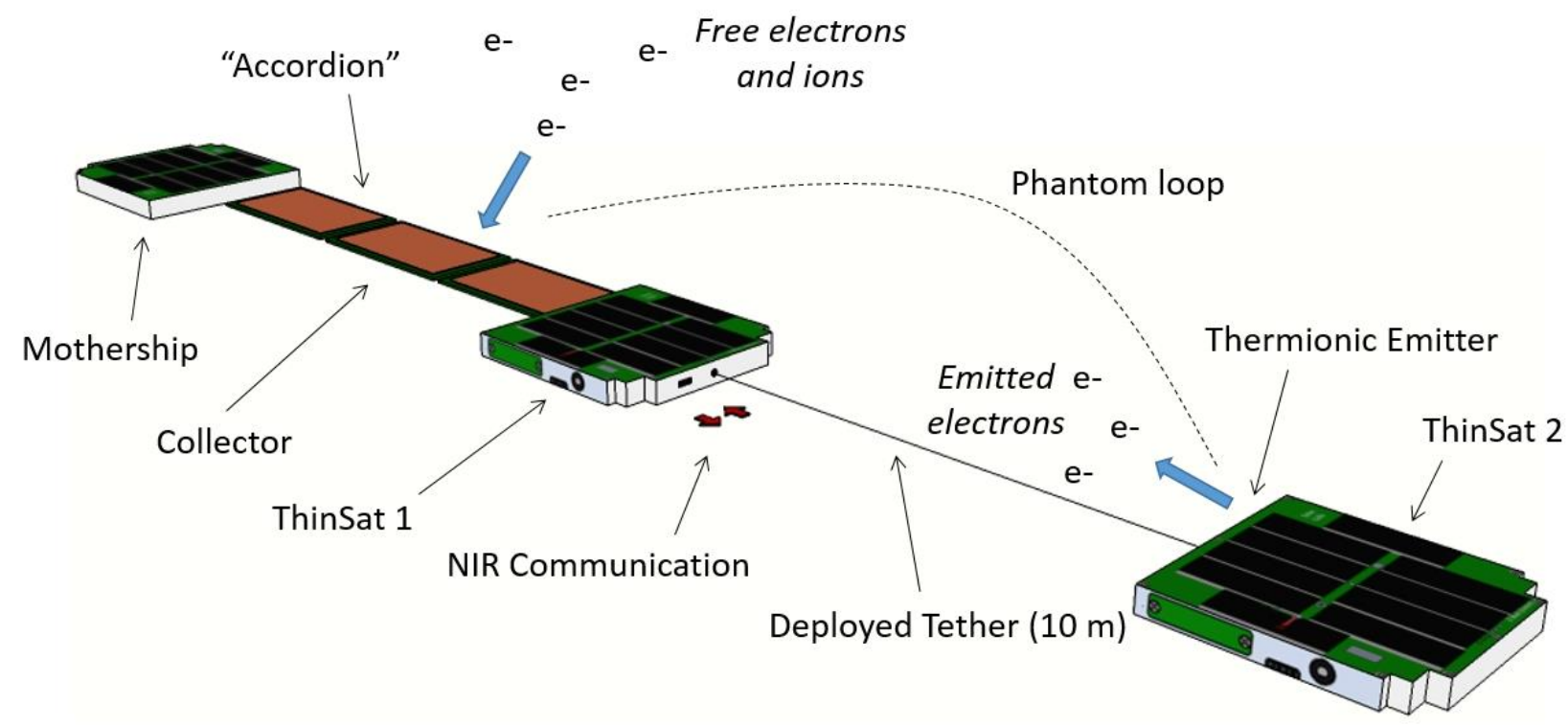

Figure 7. ThinSat System Concept.

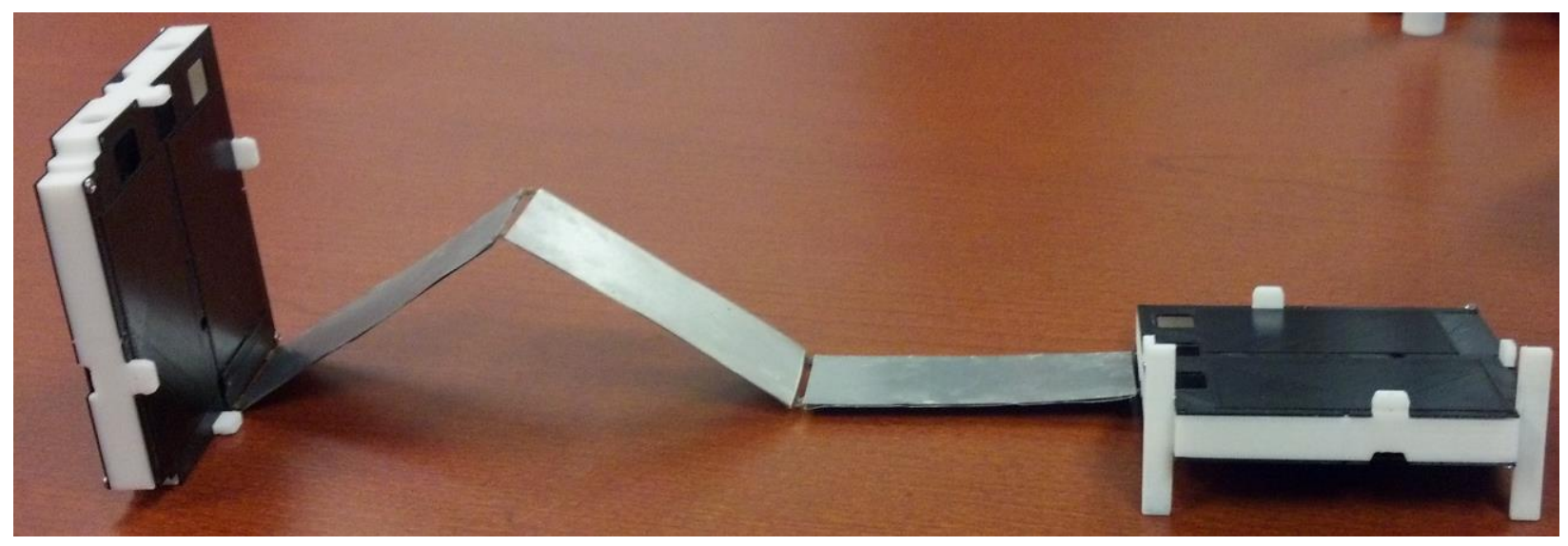

Figure 8. ThinSat and Accordion Mockup.

\subsection{System Block Diagram}

Figure 9 shows the system block diagram for ThinSat-2. ThinSat-2 acts as the master for the tethered satellites. ThinSat-2 sends tether deployment commands to ThinSat-1. ThinSat-2 also controls the phantom loop. Both satellites have simplex Globalstar radios to send data to the ground. Each satellite has its own independent power source consisting of a rechargeable battery and solar cells on both sides of the satellite. 


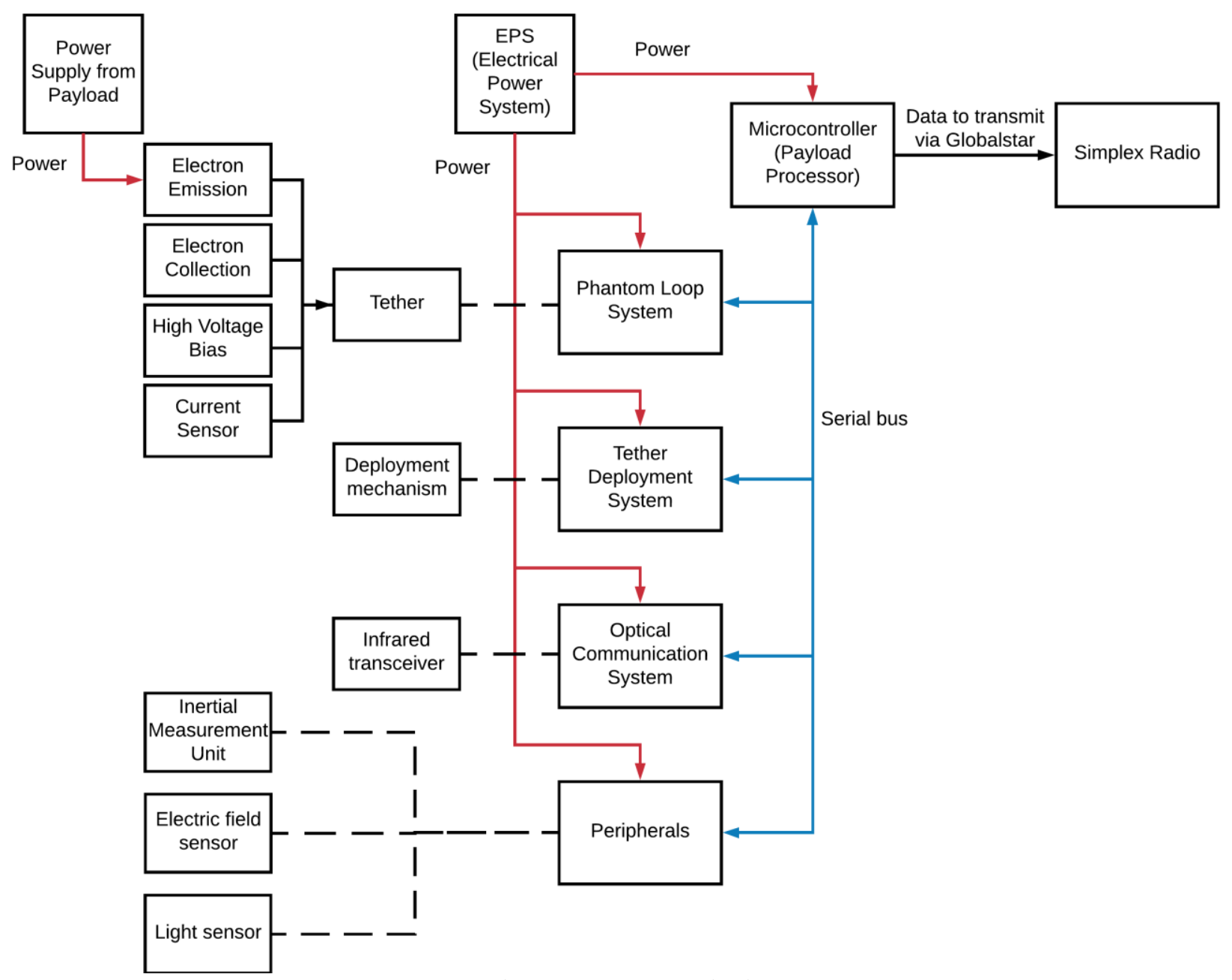

Figure 9. ThinSat-2 System Block Diagram.

\subsection{Tether Deployment Mechanism}

The tether is an insulated 0.012 " diameter super-elastic Nitinol wire. The wire is wrapped around a spool until deployment. To deploy the tether, a continuously rotating servomotor spins the spool, pushing the wire out the end of the ThinSat (Figure 10). It takes approximately 2 hours to deploy the 10-meter tether. 


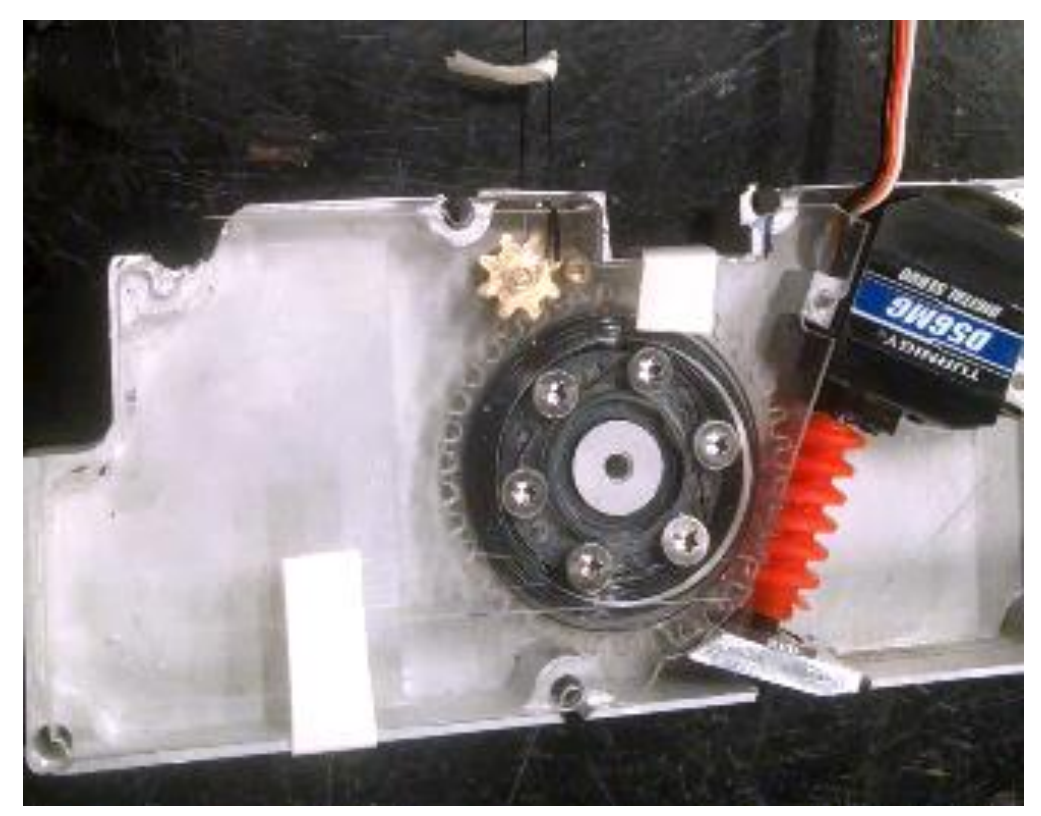

Figure 10. Tether Deployment Mechanism.

\subsection{Near-infrared (NIR) Communication Link}

Since there is no electrical connection (other than the single tether wire) between ThinSat- 1 and ThinSat-2, an optical link transfers commands and data between the satellites. We opted to use an optical link rather than radio (Bluetooth, etc.) due to the more stringent regulatory issues regarding radio signals in space.

Near infrared LED's on each satellite shine a modulated signal to optical receivers on the other satellite. The LED wavelength is $950 \mathrm{~nm}$. The modulation carrier frequency is $56 \mathrm{kHz}$. The system uses both 20-degree and 40-degree beam angles. Measured range performance is over 20 meters. 


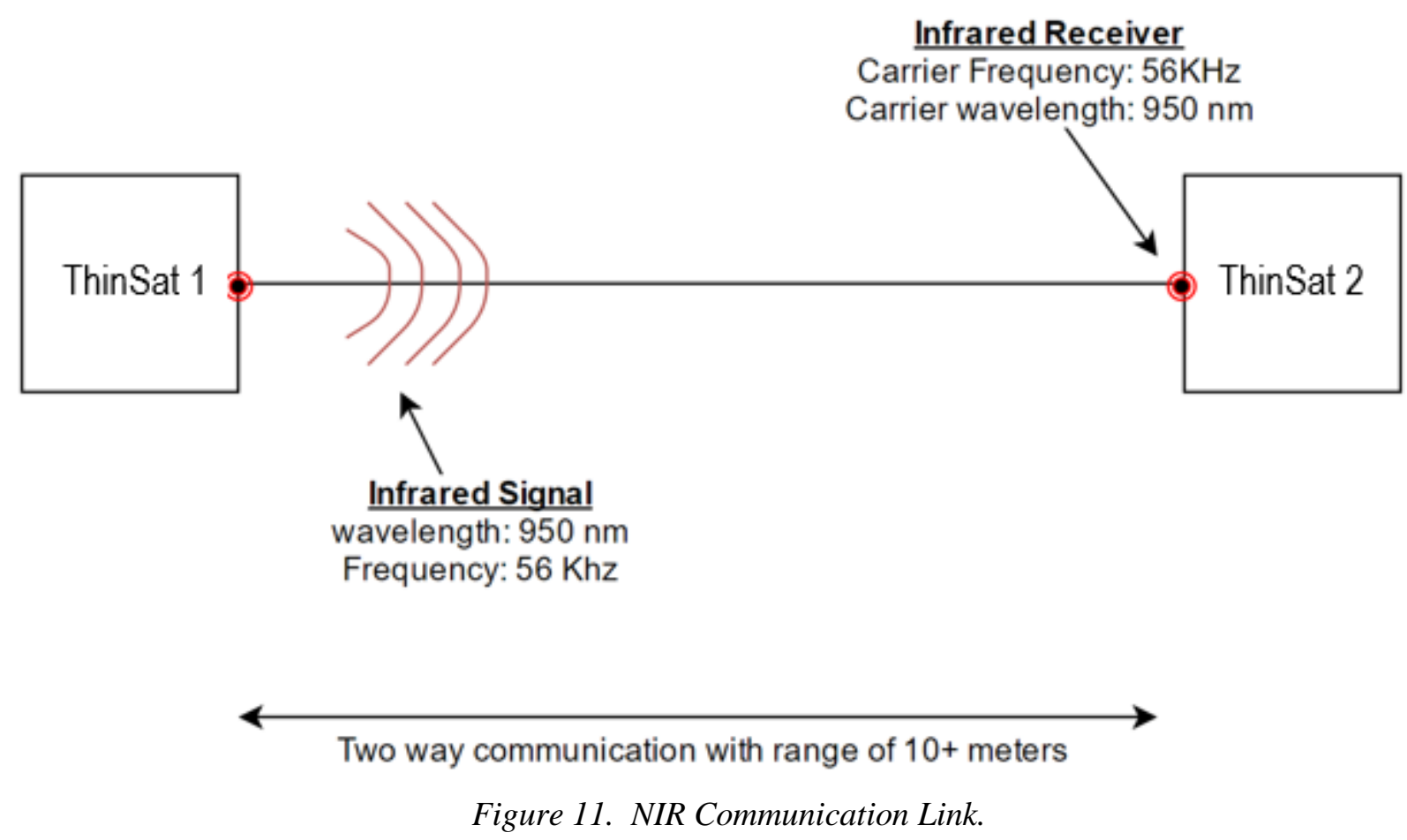

\subsection{Phantom Loop}

The phantom loop contains two primary components: a thermionic emitter and a high voltage bias supply. The thermionic emitter is a short $(\sim 2 \mathrm{~cm})$ filament of tungsten wire heated by a high current driver. When the filament temperature is high enough, the filament emits electrons. The high voltage bias supply provides the electromotive force to drive current through the phantom loop. Figure 3 in Section 5.2 above shows the phantom loop circuit.

The thermionic emitter must be located away from the satellite body to prevent electrons from flowing into the satellite instead of into the ionosphere. A mechanical emitter deployment mechanism (Figure 12) extends the emitter approximately $100 \mathrm{~mm}$ away from the satellite frame. 


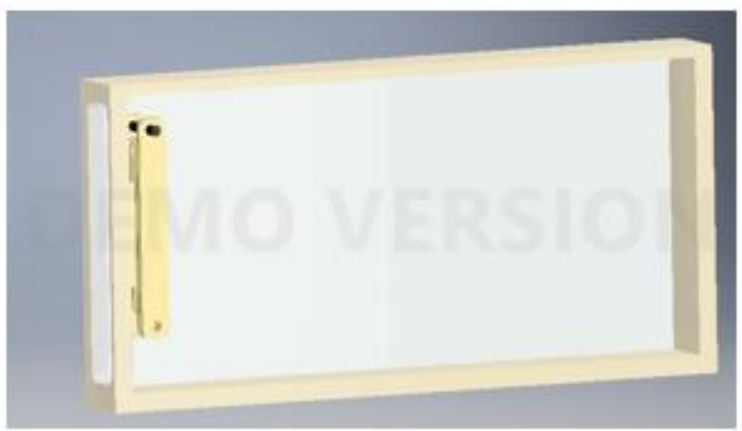

(a) Fully retracted

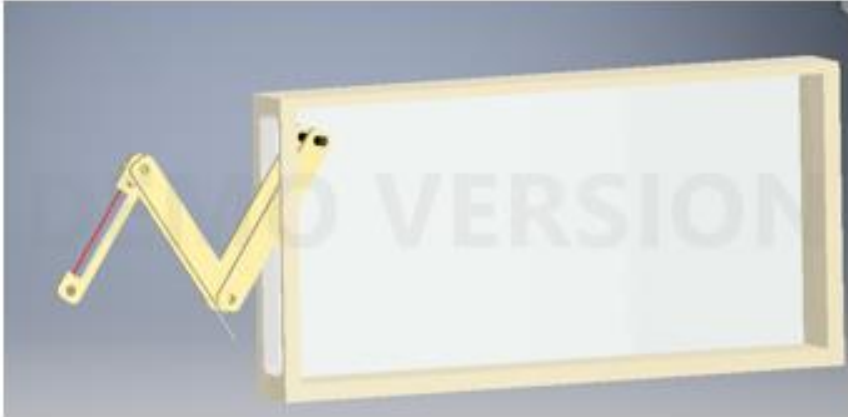

(b) Partially extended.

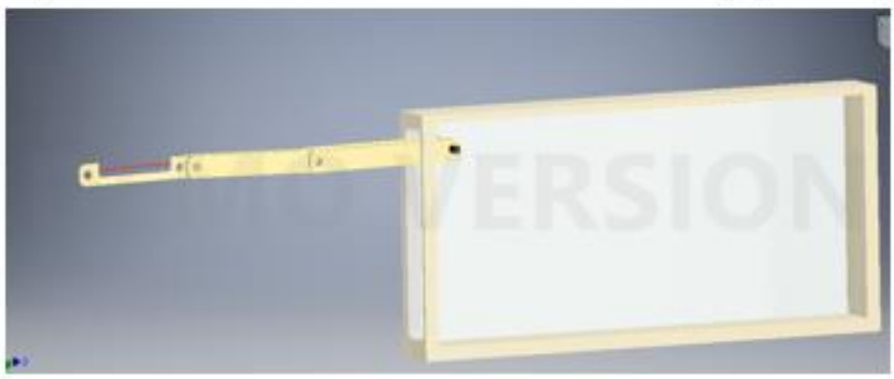

(c) Fully extended.

Figure 12. Emitter Deployment Mechanism.

\subsection{System Software}

The system software runs on the main processor, an Atmel Atmega32u4 chip. Since neither ThinSat-1 nor ThinSat-2 has a duplex radio to receive commands from the ground, the software must run autonomously. The only command received from the ground is an emergency shutdown command. This command will come from the mothership, which has a duplex radio. Figure 13 shows the system software flowchart. 


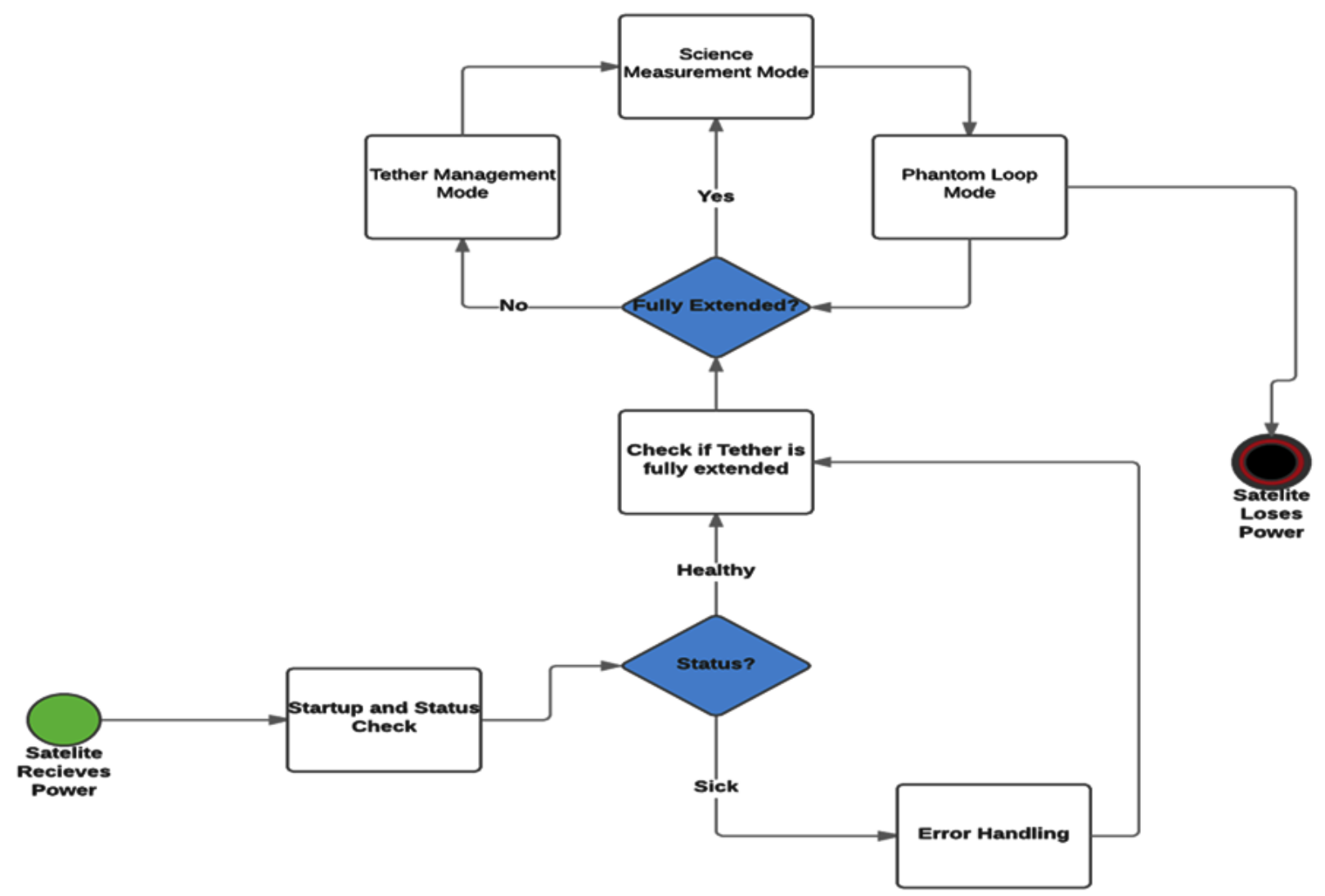

Figure 13. System Software Flowchart.

\subsection{Space Data Dashboard}

The ThinSat satellites transmit data to the ground via the Globalstar communications satellite network. Table 3 lists the data collected and sent to the ground. A web-based data dashboard receives the data and displays it in chart form. Figure 14 shows early concept examples of the displays.

Table 3. Collected Data.

\begin{tabular}{|l|l|l|}
\hline Instrument & Parameter & Range (TBR) \\
\hline Tether & Current & 0 to $5 \mathrm{~mA}$ \\
\hline Tether & Bias voltage & 0 to $200 \mathrm{~V}$ \\
\hline Collector & Bias voltage & 0 to $200 \mathrm{~V}$ \\
\hline Electric field sensor & Electric field strength & $10 \mathrm{mV}-50 \mathrm{mV}$ \\
\hline Inertial Measurement Unit & Magnetic field strength & Up to $20 \mathrm{uTesla}$ \\
\hline Inertial Measurement Unit & Acceleration & Up to $+/-2 \mathrm{~g}$ \\
\hline Light sensor & Illumination & Up to $80 \mathrm{k} l \mathrm{lux}$ \\
\hline Temperature sensor & Temperature & $-150 \mathrm{C}$ to $125 \mathrm{C}$ \\
\hline
\end{tabular}




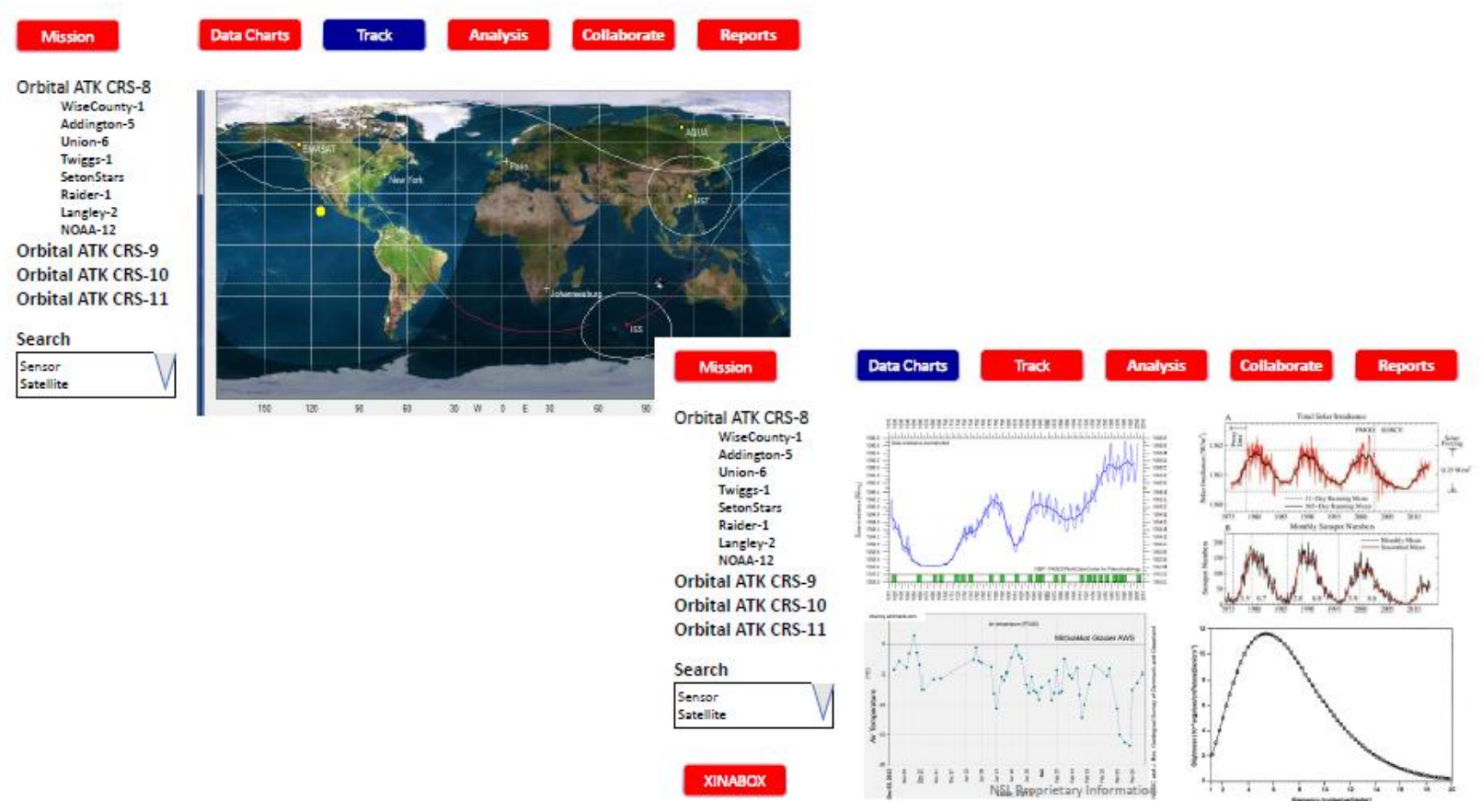

Figure 14. Data Dashboard Display - early concept design.

\subsection{Test Plan}

As stated above, the mission is to test three subsystems of the tethered satellite concept:

1) the tether deployment mechanism; 2) the high-voltage biasing to enable current flow through a "phantom loop" formed by the conductive tether and the ionosphere; and 3 ) the optical (nearinfrared) link.

\subsection{Tether Deployment Mechanism}

Without expensive equipment, the tether deployment mechanism cannot be tested under zerogravity conditions. To test the mechanism on the ground, our plan is to suspend the two satellites horizontally and validate the mechanism's ability to separate the two satellites at the desired rate. Thermal-vacuum and vibration testing will help us ensure that the mechanism will be able to survive the high-g forces experienced during a launch.

\subsection{High-voltage Biasing}

The high-voltage biasing and phantom loop circuit will undergo preliminary testing in a vacuum chamber. Since it is difficult to duplicate the ionospheric conditions (free electron/ion content in the vacuum), we will instead verify that the electrons generated by the thermionic emitter are accelerated by the bias supply between the negative and positive connectors. This will create an actual current loop rather than a phantom loop. 


\subsection{Optical Link}

We have already tested the optical data link on the ground under fluorescent ambient lighting conditions. Future testing will include flooding the area with infrared light to measure the system's signal-to-noise performance.

\subsection{Payload Circuit Board}

The full payload circuit board including the thermionic emitter will undergo thermal-vacuum and vibration testing. Items of concern include the mechanical integrity of the emitter filament and heat dissipation of the filament driver and the system microprocessor.

\subsection{Day-in-the-life Testing}

The complete two-satellite system will undergo day-in-the-life testing on the ground. This test simulates the life of the system. The test includes system initialization, executing all the modes, communication between the two satellites, transmitting data via Globalstar, etc.

\subsection{High Altitude Testing}

A high altitude balloon will carry the complete system to the edge of space $(\sim 30 \mathrm{~km}$ altitude) to test its operation. Although the launch will not subject the system to vibration, it will allow us to observe the system's operation in low temperature and low atmospheric pressure situations.

\subsection{Project Status}

As of this writing, the team has tested the Flat-Sat hardware (Figure 15) and is designing the final payload printed circuit board. Circuit board assembly and testing will take place during the spring semester. The system software has been written and tested on the Flat-Sat hardware. 


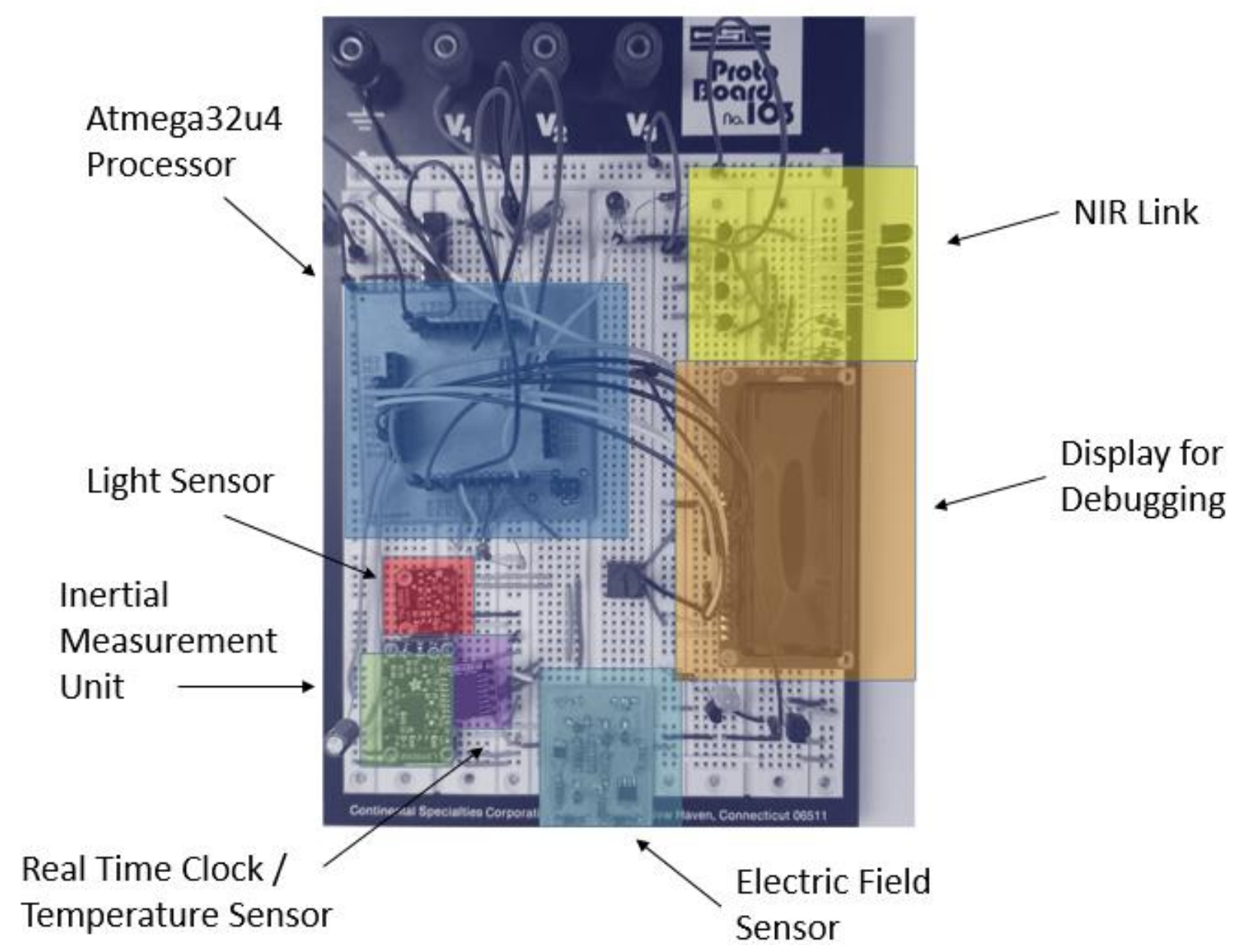

Figure 15. Flat-Sat.

\subsection{Conclusion}

For the remainder of the 2017-2018 academic year, the team will finish the ThinSat design and prepare the satellites for survival in space. Assuming everything remains on schedule, the satellites will launch into orbit on a Space Station resupply mission in October 2018.

The MagnITO-Sat and ET-Sat projects have provided excellent opportunities to expose students to space science. Through these projects, students have learned how to perform research, how to perform a rigorous system design, how to collaborate with other organizations, and how to work together as a team.

Student feedback has shown that these projects, although very challenging, have provided excellent learning opportunities for the students. Following are several excerpts from student reflection papers:

- "I have had an incredible opportunity this past semester to work with a group of Taylor students to build a satellite..."

- "I gained a lot of confidence in my presentation skills..." 
- "Some of the valuable lessons I learned while working on this project include the importance of making and sticking to a schedule, the process of developing requirements for vague tasks..."

- "Overall, this project was an incredibly valuable experience..."

- "applying concepts to lead this project has been one of the most beneficial academic endeavors I have undertaken..."

- "I learned and became more comfortable with presenting my work to an audience..."

- "When it comes to roadblocks and successes, I learned to ask the right questions and ask the right people..."

- "this class was also very difficult at times..."

- "I learned a lot about physical computing and real-life team collaboration and designing on a project..."

\subsection{Acknowledgements}

Many Taylor University students have worked on this project since its inception in 2016: Caleb Anthony, Jason Argo, Jacob Bernard, Chester Chan, David Deng, Tyler Gruthusen, Ryan Jones, Joel Kiers, Cassie Long, Micah Meleski, Neil Perry, Ben Ryker, Moriah Schlenker, Jonathan Siegelin, Justin Theien, Austin Wolgemuth, Eliza You, Edric Yu, and Joyce Yu.

The team wishes to acknowledge the many organizations and individuals who have helped the team to get to this point in the project. Organizations include the PhyXTGears Robotics Club, whose members worked as an integral part of our team to design the deployment mechanisms, Near Space Launch, Twiggs Space Lab, Virginia Space, and Tethers Unlimited. Many thanks go to Dr. Bill Chapman (formerly at Taylor University), Dr. Brian Gilchrist of the University of Michigan, Dr. Lon Enloe of the Naval Research Labs, and Dr. Rob Pfaff of NASA Goddard Space Flight Center for their advice and guidance. Finally, we are extremely grateful for the support and funding of the Indiana Space Grant Consortium.

\section{REFERENCES}

[1] Cosmo, M.L. and E.C. Lorenzini (eds.), "Tethers in Space Handbook”, Third Edition, Smithsonian Astrophysical Observatory, Cambridge, Massachusetts, USA, 1997.

[2] Sanmartin J.R., Charro M., Chen X., Lorenzini E.C., et. al. (2012). A Universal System to Deorbit Satellites at End of Life. Journal of Space Technology and Science, Vol. 26, No. 1.

[3] Carroll JA. Tether applications in space transportation. Acta Astronaut 1986. 


\section{Appendix A: Nanosatellite Space Tether Systems Requirements Specification}

\section{Overview}

\section{Mission Statement}

To design a $2 \mathrm{U}$ or $3 \mathrm{U}$ nanosatellite capable of deploying a space tether. The tether will be used to generate power as it is dragged through the ionosphere. By sending power down the tether, a magnetic force can be generated opposing the earth's magnetic field and this can be used to change the orbit.

\section{General}

3.1 The AFRL University Nanosatellite Program (UNP) User's manual dated 17 January 2016 will be used as a guideline for program management and operation.

3.2 Unless stated otherwise, all requirements in UNP9 shall be followed.

3.3 The satellite shall be designed and fabricated to be capable of launch from a Cal Poly P-POD.

3.4 The satellite shall be designed and fabricated to be capable of launch from the Planetary Systems Corporation (PSC) Canisterized Satellite Dispenser (CSD).

3.5 The Space Vehicle (SV) shall be capable to operate between $600 \mathrm{~km}$ and $150 \mathrm{~km}$ altitude.

\section{Mechanical}

4.1 All mechanical materials, weight and size requirements in the launcher shall be followed and flowed down to the payload.

4.2 The tether and tether deployment subsystem shall be inhibited from deployment prior to launch.

4.3 The tether deployment subsystem shall operate in the temperature environments experienced.

4.4 The tether shall withstand the forces from full deployment for at least 90 orbits.

4.5 The SV shall monitor temperatures of the vehicle in all representative places that dissipate power.

\section{Electrical}

5.1 All electrical materials, inhibits, power conditioning and safety requirements in the launcher shall be followed.

5.2 Power generated over the tether subsystem shall not damage or interfere with the SV electronic power supply, communications, or flight computer.

5.3 The Power subsystem shall be capable of storing power generated from the space tether in the SV batteries.

5.4 The power subsystem shall have sufficient solar power over the orbit to remain at least power neutral.

\section{Functional}

\subsection{Tether}

6.1.1 The tether shall be capable of being deployed in increments of no more than 5 meters.

6.1.2 The tether shall be capable of being retrieved without damage to the space craft.

6.1.3 The tether shall do no damage to the SV if it is damaged or broken during flight.

6.1.4 The tether shall be capable of being powered so that an opposing magnetic force is created to the Earth's magnetic force.

6.1.5 The tether shall be deployed in stages of no more than 5 meters each time.

6.1.6 The tether shall be monitored for at least 3 orbits after every staged deployment.

6.1.7 The system shall monitor the tether stress, temperature and voltages at all times. 
6.1.8 The tether shall be retrieved in no more than 5 meter stages.

6.1.9 The tether shall be retrieved if the measured stress exceeds $20 \mathrm{lb}$.

6.1.10 The tether shall be retrieved if the system believes it has broken.

6.1.11 The tether shall be retrieved if it causes instabilities to the SV that the ACS cannot correct.

6.1.12 The SV shall have the tether deployed when altitudes are under $150 \mathrm{~km}$.

\subsection{Attitude Determination and Control System}

6.2.1 The attitude control system shall be capable of maintaining position of the space vehicle within +-10 degrees (TBR) of commanded position in all 3 axis's.

6.2.2 The attitude control system shall be capable of meeting requirements within 24 hours of being powered.

6.2.3 The SV shall meet the attitude control orientation requirement prior to deploying the tether.

\subsection{Communications}

6.3.1 The SV shall be capable of sending and receiving data over the Globalstar satellite communication network.

6.3.2 The SV shall be capable of communicating with the network no less than $65 \%$ of the time during the mission.

6.3.3 The SV shall not communicate for the first 45 minutes of free flight.

6.3.4 The SV shall transmit health information no less than once per orbit.

6.3.5 The Health information shall consist of temperature data, voltages and status and mode.

6.3.6 When the SV is under 150KM altitude, it shall transmit health and tether data every 5 seconds.

\subsection{Safe Mode}

6.4.1 The SV shall have a Safe mode in case of power faults or system failures.

6.4.2 The SV shall exit Safe mode only when electrical power characteristics are within specification. 


\section{Appendix B: ThinSat Requirements}

1. The payload shall comply with the ET-Sat mass, center of gravity, and size constraints.

2. The payload shall operate on $5 \mathrm{~V}$ and $3.3 \mathrm{~V}$ rails.

3 . The payload current shall not exceed $100 \mathrm{~mA}$ continuous.

4. The payload shall measure a magnetic field of up to 30 micro-Tesla.

5. The payload should be able to measure electric field strength of $\sim-100 \mathrm{mV} / \mathrm{m}$ to $100 \mathrm{mV} / \mathrm{m}$ with the capability to withstand spikes up to $1.5 \mathrm{~V} / \mathrm{m}$.

6. The payload shall house a tether deployment mechanism.

7. The tether shall be capable of deployment in specific increments.

8. The tether deployment system shall be able to measure strain up to $15 \mathrm{~N} \mathrm{w} / \pm 0.1 \mathrm{~N}$ resolution in three axes.

9. The payload shall implement and test a phantom loop.

10. The payload shall measure tether current of 1 to $10 \mathrm{~mA}$.

11. The payload shall measure tether voltage of 0 to $+200 \mathrm{~V}$.

12. The payload shall measure spacecraft bias voltage of 0 to $-200 \mathrm{~V}$.

13. The payload shall measure spacecraft temperature of $-150 \mathrm{C}$ to $125 \mathrm{C}$.

14. The payload shall measure ambient illumination of visible and near-infrared wavelengths and intensity.

15. The payload shall include an inertial measurement unit.

16. The satellite should be able to operate without attitude control. 